\title{
Superconductivity in the Orbital Degenerate Model for Heavy Fermion Systems
}

\author{
Tetsuya Takimoto†, Takashi Hotta†, and Kazuo Uedał† \\ $\dagger$ Advanced Science Research Center, Japan Atomic Energy Research Institute, Tokai, Ibaraki 319-1195, Japan \\ $\ddagger$ Institute for Solid State Physics, University of Tokyo, 5-1-5 Kashiwa-no-ha, Kashiwa, Chiba 277-8581, Japan
}

(November 8, 2018)

\begin{abstract}
Magnetism and superconductivity of new heavy fermion compounds $\mathrm{CeTIn}_{5}$ ( $\mathrm{T}=\mathrm{Co}$, Rh and Ir) are investigated by applying fluctuation exchange approximation to an orbital degenerate Hubbard model. The superconducting phase with $d_{x^{2}-y^{2}}$-symmetry is found to appear next to the antiferromagnetic phase with increasing the orbital splitting energy. The present theory suggests that the orbital splitting energy plays a key role of controlling parameter for the quantum phase transitions in the heavy fermion system.
\end{abstract}

\section{INTRODUCTION}

Superconductivity in strongly correlated electron systems has been one of central issues in the research field of condensed matter physics, since the pioneering discovery of superconductivity in $\mathrm{CeCu}_{2} \mathrm{Si}_{2}$ [1]. The subsequent discovery of high temperature superconductivity in cuprates has accelerated further investigations of this subject, leading to unambiguous identification of the unconventional nature of $d_{x^{2}-y^{2}}$-wave pairing in cuprates. Recently, new heavy fermion compounds $\mathrm{CeTIn}_{5}(\mathrm{~T}=\mathrm{Rh}$, Ir, and Co) have been discovered [2]. Among them, $\mathrm{CeRhIn}_{5}$ exhibits an antiferromagnetic (AFM) transition at a Néel temperature $T_{\mathrm{N}}=3.8 \mathrm{~K}$ and becomes superconducting only under hydrostatic pressure larger than 15 kbar. On the other hand, both $\mathrm{CeIrIn}_{5}$ and $\mathrm{CeCoIn}_{5}$ are superconducting at ambient pressure with transition temperatures $T_{\mathrm{c}}=0.4 \mathrm{~K}$ and $2.3 \mathrm{~K}$, respectively. Especially, the $T_{\mathrm{c}}$ of $\mathrm{CeCoIn}_{5}$ is the highest among those yet observed for heavy fermion superconductors [3].

Reflecting the fact that $\mathrm{CeTIn}_{5}$ has the HoCoGa 5 -type tetragonal crystal structure, quasi two-dimensional Fermi surfaces have been observed in de Haas-van Alphen experiments for the compounds, consistent with the bandstructure calculation results [4]. Concerning the superconducting state, nuclear relaxation rate in $\mathrm{CeTIn}_{5}$ exhibits $T^{3}$ behavior below $T_{\mathrm{c}}$ [5] and thermal conductivity in $\mathrm{CeCoIn}_{5}$ is found to include a component with four-fold symmetry [6, strongly suggesting $d_{x^{2}-y^{2}}$-wave pairing in the superconducting phase of $\mathrm{CeTIn}_{5}$. Furthermore, it has been shown that in the alloy system $\mathrm{CeRh}_{1-x} \operatorname{Ir}_{x} \operatorname{In}_{5}$, the superconducting phase appears in the neighborhood of the AFM phase [7]. A natural consequence of these experimental results is that superconductivity in $\mathrm{CeTIn}_{5}$ compounds is induced by AFM spin fluctuations, similarly to high- $T_{\mathrm{c}}$ superconductivity.

In spite of the similarities mentioned above, here we should emphasize several differences between heavy fermion superconductors such as $\mathrm{CeTIn}_{5}$ and high- $T_{\mathrm{c}}$ cuprates. First of all, relevant electrons in high- $T_{\mathrm{c}}$ superconductors are almost itinerant $3 d$-electrons, while for heavy fermion superconductors, relevant ones are $f$ electrons, which almost localized and their dispersion is mainly determined by hybridization with conduction electrons. Another important difference is concerned with the electronic states relevant to low-energy physics. For cuprates, it is widely recognized that a single-band model for holes in $d_{x^{2}-y^{2}}$ orbitals on the square lattice is a good starting point. On the other hand, for heavy fermion superconductors, several Fermi surfaces are observed in general. Such complex electronic states may be eventually traced back to the orbital degeneracy and relatively weak crystalline electric field (CEF) effect of $f$-electrons compared with the $3 d$-electrons in the $\mathrm{CuO}_{2}$ plane. It implies that construction of a realistic microscopic model is not an easy work for heavy fermion systems. Therefore, up to now, most of theoretical investigations for superconductivity in heavy fermion systems have been restricted in a phenomenological level.

In this paper, we discuss the effect of orbital fluctuations on superconductivity based on a microscopic theory applied to a microscopic model. In the next section, we introduce an orbital degenerate model obtained including important characters of $\mathrm{CeTIn}_{5}$. Then, in order to study the superconducting transition induced by spin and/or orbital fluctuations, we develop a strong-coupling theory using fluctuation exchange (FLEX) approximation 8] in which spin and orbital fluctuations as well as the single-particle spectrum are determined self-consistently. Finally, we discuss experimental results for $\mathrm{CeTIn}_{5}$ in the light of the present theory.

\section{MODEL HAMILTONIAN}

In order to introduce a minimal model for $f$-electron systems, we start from the local basis for a $\mathrm{Ce}^{3+}$ ion. Among 14-fold degenerate $4 f$-electronic states, due to the effect of strong spin-orbit coupling, only the $j=5 / 2$ sextet effectively contributes to the low-energy excitations ( $j$ is total angular momentum). Under the cubic CEF, this sextet is further split into $\Gamma_{7}$ doublet and $\Gamma_{8}$ quartet, where the corresponding eigen-states are given by $\left|\Gamma_{7 \pm}\right\rangle=\sqrt{1 / 6}| \pm 5 / 2\rangle-\sqrt{5 / 6}|\mp 3 / 2\rangle,\left|\Gamma_{8 \pm}^{(1)}\right\rangle=$ $\sqrt{5 / 6}| \pm 5 / 2\rangle+\sqrt{1 / 6}|\mp 3 / 2\rangle$, and $\left|\Gamma_{8 \pm}^{(2)}\right\rangle=| \pm 1 / 2\rangle$. Here + and - in the subscripts denote "pseudo-spin" up and 
down in each Kramers doublet, respectively. For simplicity, we consider only $\left|\Gamma_{8}\right\rangle$ states, further split into two Kramers doublets as $\left|\Gamma_{8 \pm}^{(1)}\right\rangle$ and $\left|\Gamma_{8 \pm}^{(2)}\right\rangle$ under the additional tetragonal CEF. Note that $\left|\Gamma_{8 \pm}^{(1)}\right\rangle$ and $\left|\Gamma_{8 \pm}^{(2)}\right\rangle$ belong to $\Gamma_{7}$ and $\Gamma_{6}$ irreducible representations, respectively, in the tetragonal system. Validity of this assumption for $\mathrm{CeTIn}_{5}$ will be discussed later in this paper, but here we stress that the Hamiltonian constructed from $\Gamma_{8}$ quartet is the simplest model including essential physics of interplay between spin and orbital fluctuations.

In order to consider itinerant features of $4 f$-electrons, we take into account nearest-neighbor hopping of $f$ electrons by the tight-binding method [9]. It should be noted that the matrix elements of the nearest-neighbor hopping depend on not only the orbital but also the hopping direction, since the forms of wavefunctions of $\left|\Gamma_{8 \pm}^{(1)}\right\rangle$ and $\left|\Gamma_{8 \pm}^{(2)}\right\rangle$ states are different from each other. We can determine the hopping matrix elements by assuming that the overlap integral through the $\sigma$-bond $(f f \sigma)$ is dominant. Since $\mathrm{CeTIn}_{5}$ has a tetragonal crystal structure and quasi two-dimensional Fermi surfaces have been experimentally observed [4, 10], it is natural to consider the two-dimensional square lattice composed of $\mathrm{Ce}^{3+}$ ions. Although the hybridization of $f$ electrons with In $5 p$ electronic states may be the main source of kinetic energy for $f$ electrons, in the present scheme such an effect is considered as the effective hopping amplitude for $f$ quasiparticles, after the $p$-electron degrees of freedom are integrated out.

By further adding the on-site Coulomb interaction terms among $f$-electrons, the effective Hamiltonian with orbital degeneracy is given by

$$
\begin{aligned}
H & =\sum_{\mathbf{i} \mathbf{a} \tau \tau^{\prime} \sigma} t_{\tau \tau^{\prime}}^{\mathbf{a}} f_{\mathbf{i} \tau \sigma}^{\dagger} f_{\mathbf{i}+\mathbf{a} \tau^{\prime} \sigma}-\varepsilon \sum_{\mathbf{i}}\left(n_{\mathbf{i} 1 \sigma}-n_{\mathbf{i} 2 \sigma}\right) / 2 \\
& +U \sum_{\mathbf{i} \tau} n_{\mathbf{i} \tau \uparrow} n_{\mathbf{i} \tau \downarrow}+U^{\prime} \sum_{\mathbf{i} \sigma \sigma^{\prime}} n_{\mathbf{i} 1 \sigma} n_{\mathbf{i} 2 \sigma^{\prime}}
\end{aligned}
$$

where $f_{\mathbf{i} \tau \sigma}$ is the annihilation operator for an $f$-electron with pseudo-spin $\sigma$ in the $\tau$-orbital state $\Gamma_{8}^{(\tau)}$ at site $\mathbf{i}$, $\mathbf{a}$ is the vector connecting nearest-neighbor sites, and $n_{\mathbf{i} \tau \sigma}=f_{\mathbf{i} \tau \sigma}^{\dagger} f_{\mathbf{i} \tau \sigma}$. The first term represents the nearestneighbor hopping of $f$-electrons with the amplitude $t_{\tau \tau^{\prime}}^{\mathbf{a}}$ between $\tau$ and $\tau^{\prime}$ orbitals along the $\mathbf{a}$-direction, given by $t_{11}^{\mathrm{x}}=-\sqrt{3} t_{12}^{\mathrm{x}}=-\sqrt{3} t_{21}^{\mathrm{x}}=3 t_{22}^{\mathrm{x}}=1$ for $\mathbf{a}=\mathbf{x}$ and $t_{11}^{\mathbf{y}}=\sqrt{3} t_{12}^{\mathbf{y}}=$ $\sqrt{3} t_{21}^{\mathrm{y}}=3 t_{22}^{\mathrm{y}}=1$ for $\mathbf{a}=\mathbf{y}$, respectively, in energy units where $t_{11}^{\mathrm{x}}=1$. The second term denotes the tetragonal $\mathrm{CEF}$, leading to an energy splitting $\varepsilon$ between the two orbitals. In the third and fourth terms, $U$ and $U^{\prime}$ are the intra- and inter-orbital Coulomb interactions, respectively. Due to the rotational invariance in the orbital space for the interaction part, $U^{\prime}$ should be equal to $U$, when we ignore the Hund's rule coupling, since it is irrelevant in the large- $U$ limit for the quarter-filling case with one $f$-electron per site. Thus, in this paper, we restrict ourselves to the case of $U=U^{\prime}$. Note also that in the quarter-filling case, the present model is virtually reduced to the half-filled single-orbital Hubbard model in the limit of $\varepsilon=\infty$.

\section{FLEX APPROXIMATION}

In our previous work, we have developed a weakcoupling theory for superconductivity based on the same orbital degenerate model described above, using the static spin and orbital fluctuations obtained within the random phase approximation (RPA) [11]. Various superconducting phase have been found around varieties of ordered phases, whose boundaries are determined by the RPA instability. In order to develop more sophisticated theory, we should include effects of (1) mode-mode coupling and (2) quasi-particle damping, neglected in the previous work. Regarding (1), the RPA does not incorporate effects of mode-mode coupling between fluctuations, but the mode-mode coupling modifies significantly the temperature and frequency dependences of spin and orbital fluctuations. Concerning (2), within the weakcoupling theory for superconductivity, damping of quasiparticles by the scattering due to spin and orbital fluctuations is not taken into account, which leads to the suppression of superconductivity. Therefore, the weakcoupling theory generally overestimates the region of superconducting phase.

In the present paper, we apply the fluctuation exchange (FLEX) approximation [8] to the orbital degenerate model discussed in the preceding section. The FLEX approximation has the following two features: (1) This is a kind of the mode-mode coupling theory, where spin and orbital fluctuations and the spectra of $f$-electrons are self-consistently determined through the fluctuation exchange self-energy. (2) It provides the Dyson-Gorkov equation where the normal and anomalous self-energies are obtained on an equal footing. Here we emphasize that the FLEX approximation has been successful to understand consistently the normal and superconducting states of high- $T_{\mathrm{c}}$ cuprates, in particular with reasonable estimation of $T_{\mathrm{c}} 12$. Therefore, it is interesting to apply the FLEX approximation to the orbital degenerate model to understand its properties concerning superconductivity.

In the doubly degenerate case, the Green's functions for $f$-electrons form a $2 \times 2$ matrix and they follow the Dyson-Gorkov equations

$$
\hat{G}(k)=\hat{G}^{(0)}(k)+\hat{G}^{(0)}(k) \hat{\Sigma}^{(1)}(k) \hat{G}(k),
$$

where $\hat{G}^{(0)}(k)$ is a matrix for the non-interacting Green's function. Within the FLEX approximation, components of the self-energy matrix $\Sigma_{m l}^{(1)}(k)$ are given by

$$
\Sigma_{m l}^{(1)}(k)=\frac{T}{N_{0}} \sum_{q} \sum_{\mu \nu} V_{\mu m, \nu l}^{\mathrm{eff}}(q) G_{\mu \nu}(k-q),
$$

with 


$$
\begin{aligned}
& V_{\mu m, \nu l}^{\mathrm{eff}}(q)=\left[\frac{3}{2} \hat{U}^{\mathrm{s}} \hat{\chi}^{\mathrm{s}}(q) \hat{U}^{\mathrm{s}}+\frac{1}{2} \hat{U}^{\mathrm{o}} \hat{\chi}^{\mathrm{o}}(q) \hat{U}^{\mathrm{o}}\right. \\
& \left.-\frac{1}{4}\left(\hat{U}^{\mathrm{s}}+\hat{U}^{\mathrm{o}}\right) \hat{\bar{\chi}}(q)\left(\hat{U}^{\mathrm{s}}+\hat{U}^{\mathrm{o}}\right)+\frac{3}{2} \hat{U}^{\mathrm{s}}-\frac{1}{2} \hat{U}^{\mathrm{o}}\right]_{\mu m, \nu l},
\end{aligned}
$$

where the first and second terms of $V_{\mu m, \nu l}^{\mathrm{eff}}(q)$ give contributions of the spin and orbital fluctuations, respectively, to the self-energy.

In Eq. (4), $\hat{\chi}^{\mathrm{s}}(q)$ and $\hat{\chi}^{\mathrm{O}}(q)$ are the $4 \times 4$ matrices of the spin and orbital fluctuations, given by

$$
\begin{aligned}
& \hat{\chi}^{\mathrm{s}}(q)=\left[\hat{1}-\hat{U}^{\mathrm{s}} \hat{\bar{\chi}}(q)\right]^{-1} \hat{\bar{\chi}}(q), \\
& \hat{\chi}^{\mathrm{o}}(q)=\left[\hat{1}+\hat{U}^{\mathrm{o}} \hat{\bar{\chi}}(q)\right]^{-1} \hat{\bar{\chi}}(q),
\end{aligned}
$$

where the matrix element of the irreducible susceptibility $\bar{\chi}_{i j, s t}(q)$ is given by

$$
\bar{\chi}_{i j, s t}(q)=-\frac{T}{N_{0}} \sum_{k} G_{s i}(k+q) G_{j t}(k),
$$

For the susceptibility matrices, the labels of row and column appear in the order $11,22,12$, and 21 , pairs of orbital indices 1 and 2 . In these expressions, $T$ is a temperature, $N_{0}$ is number of unit cells, abbreviations $k=\left(\mathbf{k}, i \omega_{n}\right)$ and $q=\left(\mathbf{q}, i \Omega_{n}\right)\left(\omega_{n}=(2 n+1) \pi T, \Omega_{n}=2 n \pi T\right)$ are used. The interaction matrices $\hat{U}^{\mathrm{s}}$ and $\hat{U}^{\mathrm{o}}$ are given by

$$
\hat{U}^{\mathrm{s}}=\left[\begin{array}{cccc}
U & 0 & 0 & 0 \\
0 & U & 0 & 0 \\
0 & 0 & U^{\prime} & 0 \\
0 & 0 & 0 & U^{\prime}
\end{array}\right], \quad \hat{U}^{\mathrm{o}}=\left[\begin{array}{cccc}
U & 2 U^{\prime} & 0 & 0 \\
2 U^{\prime} & U & 0 & 0 \\
0 & 0 & -U^{\prime} & 0 \\
0 & 0 & 0 & -U^{\prime}
\end{array}\right] .
$$

By solving the self-consistent equations, the spin and orbital fluctuations and the Green's functions for $f$ electrons are determined simultaneously.

In order to discuss superconductivity, it is necessary to calculate anomalous self-energy. The matrix element of the anomalous self-energy $\Sigma_{m l}^{(2)}(k)$ is obtained by the functional derivative of the thermodynamic potential with respect to the anomalous Green's function as follows

$$
\Sigma_{m l}^{(2)}(k)=\frac{T}{N_{0}} \sum_{q} \sum_{\mu \nu} V_{\mu m, l \nu}^{\xi}(q) F_{\mu \nu}(k-q),
$$

with

$$
\hat{F}(k)=\hat{G}(k) \hat{\Sigma}^{(2)}(k) \hat{G}(-k),
$$

where the matrix elements of the effective pairing interactions for spin-singlet and spin-triplet channels are given, respectively, by

$$
\begin{aligned}
V_{\mu m, l \nu}^{\mathrm{S}}(q)= & {\left[-\frac{3}{2} \hat{U}^{\mathrm{s}} \hat{\chi}^{\mathrm{S}}(q) \hat{U}^{\mathrm{s}}+\frac{1}{2} \hat{U}^{\mathrm{o}} \hat{\chi}^{\mathrm{o}}(q) \hat{U}^{\mathrm{o}}\right.} \\
& \left.-\frac{1}{2}\left(\hat{U}^{\mathrm{s}}+\hat{U}^{\mathrm{o}}\right)\right]_{\mu m, l \nu}, \\
V_{\mu m, l \nu}^{\mathrm{T}}(q)= & {\left[\frac{1}{2} \hat{U}^{\mathrm{s}} \hat{\chi}^{\mathrm{S}}(q) \hat{U}^{\mathrm{s}}+\frac{1}{2} \hat{U}^{\mathrm{o}} \hat{\chi}^{\mathrm{o}}(q) \hat{U}^{\mathrm{o}}\right.} \\
- & \left.\frac{1}{2}\left(\hat{U}^{\mathrm{s}}+\hat{U}^{\mathrm{o}}\right)\right]_{\mu m, l \nu} .
\end{aligned}
$$

The $T_{\mathrm{c}}$ is obtained by the temperature at which the maximum eigenvalue of Eq. (8) becomes unity. As already pointed out in the previous works based on the RPA [13, 11, one can see from these effective pairing interactions that developments of both spin and orbital fluctuations have a destructive interference for the singlet channel, while they are constructive for the triplet one.

\section{CALCULATED RESULTS}

The FLEX calculation is numerically carried out for each value of $\varepsilon$ at fixed parameter values of $U=U^{\prime}=4$ and $n=1$ corresponding to one $f$-electron density per site. All summations involved in the above self-consistent equations are performed using the fast Fourier transformation algorithm for the $\mathbf{k}$-space with $32 \times 32$ meshes in the first Brillouin zone and for Matsubara frequency sum with energy cut-off five times larger than the relevant band width. In Fig. 1, q dependences of the principal components of $\hat{\chi}^{\mathrm{s}}(\mathbf{q}, 0)$ and $\hat{\chi}^{\mathrm{O}}(\mathbf{q}, 0)$ are shown for a fixed temperature $T=0.02$ for different level splitting, $\varepsilon$ : The upper panel for $\varepsilon=0$ and the lower panel for $\varepsilon=2$. For $\varepsilon=0$, corresponding to the orbitally degenerate case, the AFM spin fluctuation in the $\tau=1$ orbital is enhanced, but not sufficiently developed to induce $d_{x^{2}-y^{2}}$-wave superconductivity. With increasing the orbital splitting energy to $\varepsilon=2$, the AFM spin fluctuation for the $\tau=1$ orbital further develops, and orbital fluctuations are completely suppressed, in comparison with the developed AFM spin fluctuation.

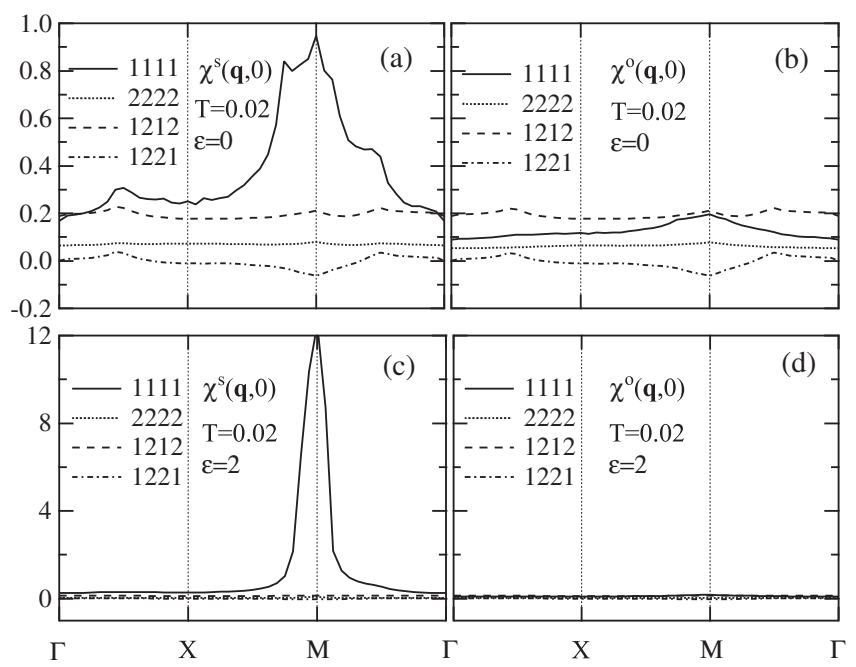

FIG. 1. (a) Spin and (b) orbital susceptibilities in $\mathbf{q}$ space for $\varepsilon=0$. (c) and (d) are for $\varepsilon=2$.

In Fig. 2, the phase diagram obtained within the FLEX approximation for the orbital degenerate system are shown, where the solid and open circles describe the superconducting and AFM transitions, respectively. The dotted curve is a schematic phase boundary expected between the two phases. From Fig. 2, we can 
see that (1) the spin-singlet superconducting phase with $B_{1 \mathrm{~g}}$-symmetry appears next to the AFM phase and (2) $T_{\mathrm{c}}$ is enhanced with increasing the orbital splitting energy $\varepsilon$. From these observations, we can conclude that the superconducting phase is induced by the development of the AFM spin fluctuations for the $\tau=1$ orbital with increasing the orbital splitting energy $\varepsilon$.

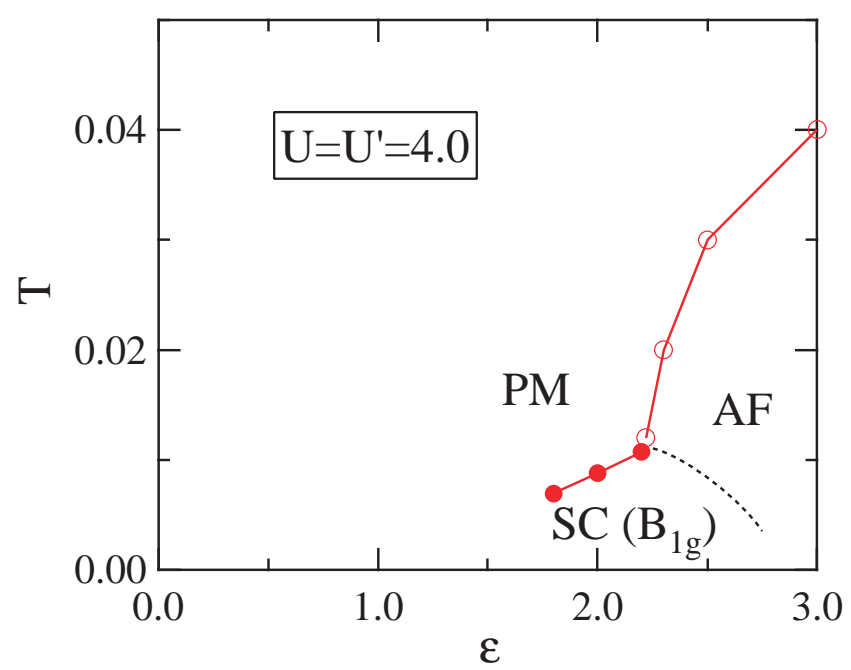

FIG. 2. Phase diagram in the $T-\varepsilon$ plane for $U=U^{\prime}=4.0 \mathrm{ob}-$ tained by the FLEX approximation.

\section{SUMMARY AND DISCUSSION}

Let us now discuss the experimental results for $\mathrm{CeTIn}_{5}$ in comparison with the present theoretical results. First we should note that in the actual tetragonal crystal, the four-fold degenerate $\Gamma_{8}$ states in the cubic notation split into two Kramers doublets, $\Gamma_{7}$ and $\Gamma_{6}$, as we mentioned in the Sec. 2. Therefore, in the tetragonal system, the $j=5 / 2$ states split into two $\Gamma_{7}$ and one $\Gamma_{6}$. Analyses of experimental data of magnetic susceptibility of $\mathrm{CeTIn}_{5}$ by using the CEF theory seem to be consistent more with the level scheme where two $\Gamma_{7}$ are lower than the $\Gamma_{6}$ [14. The energy splitting between the two $\Gamma_{7}$ is estimated as $68 \mathrm{~K}$ for $\mathrm{CeRhIn}_{5}, 61 \mathrm{~K}$ for $\mathrm{CeIrIn}_{5}$, and $151 \mathrm{~K}$ for $\mathrm{CeCoIn}_{5}$. According to the present analysis, higher $T_{\mathrm{c}}$ is obtained for larger $\varepsilon$, consistent with the tendency in $T_{\mathrm{c}}$ of the two superconducting materials: $T_{\mathrm{c}}=0.4 \mathrm{~K}$ for $\mathrm{CeIrIn}_{5}$ and $T_{\mathrm{c}}=2.3 \mathrm{~K}$ for $\mathrm{CeCoIn}_{5}$.

However, the antiferromagnetically ordered $\mathrm{CeRhIn}_{5}$ with $T_{\mathrm{N}}=3.8 \mathrm{~K}$ has an intermediate value for $\varepsilon$. One possible scenario to understand the discrepancy is to consider difference of quasi-two dimensionality, as we have pointed out in [11]. To make more direct and quantitative comparison with experimental results, however, one should be aware of an assumption for the model used in the present study, which may be called as $\Gamma_{8}$ model. Namely, this $\Gamma_{8}$ model assumes that $\Gamma_{7}$ and $\Gamma_{6}$ are the lower two Kramers doublets, which may be different from the level scheme obtained from the experiments. Thus, it will be an interesting future problem to elucidate the role of orbital splitting for the more realistic two $\Gamma_{7}$ model, which includes not only the nearest neighbor hopping but also the next nearest neighbor one and thus, may reproduce the realistic electronic states better than the $\Gamma_{8}$ model [15].

In order to construct a whole story for $\mathrm{CeTIn}_{5}$ compounds, it may be required to use the even more realistic $f$ - $p$ model including the $f$ - $p$ hybridization explicitly. In the $f$ - $p$ model, we can have two different energy scales: The large energy scale is the band width for the conduction electron and/or the Coulomb interaction, while the small energy scale corresponds to the energy splitting of the CEF levels discussed here. The present study indicates the possibility that the small energy scale of the CEF level splitting plays a key role as the controlling parameter of quantum phase transitions.

In summary, based on the effective microscopic model with orbital degeneracy for $f$-electron systems, we have proposed that the orbital splitting energy is the parameter controlling the change from the paramagnetic to the

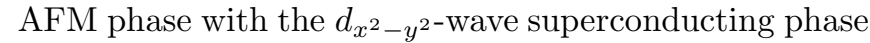
in between. Actually, the self-consistent FLEX approximation applied to the orbital degenerate model shows that the $d_{x^{2}-y^{2}}$-wave superconducting phase is induced by increasing the orbital splitting energy.

\section{ACKNOWLEDGEMENT}

The authors would like to thank T. Maehira for many helpful discussions. T.H. and K.U. are supported by the Grant-in-Aid for Scientific Research from Japan Society for the Promotion of Science.

\section{REFERENCES}

[1] Steglich F et al. 1979 Phys. Rev. Lett. 431892

[2] Hegger H et al. 2000 Phys. Rev. Lett. 844986

Petrovic C et al. 2001 Europhys. Lett. 53354

Petrovic C et al. 2001 J. Phys.: Condens. Matter 13 L337

[3] Recently it has been discovered that $\mathrm{PuCoGa}_{5}$ exhibits superconductivity with $T_{\mathrm{c}}=18.5 \mathrm{~K}$. See Sarrao J L et al. 2002 Nature(London) 420297

[4] Haga Y et al. 2001 Phys. Rev. B 63 R060503

[5] Kohori Y et al. 2000 Eur. Phys. J. B 18601

Zheng G-q et al. 2001 Phys. Rev. Lett. 864664

[6] Izawa K et al. 2001 Phys. Rev. Lett. 87057002

[7] Pagliuso P G et al. 2001 Phys. Rev. B 64 R100503

[8] Bickers N E et al. 1989 Phys. Rev. Lett. 62961 
[9] Maehira T et al. 2002 J. Phys. Soc. Jpn. Suppl. A 71285 and see also Maehira $\mathrm{T}$ et al. cond-mat/0211360

[10] Settai R et al. 2001 J. Phys.: Condens. Matter. 13 L627

[11] Takimoto T et al. 2002 J. Phys.: Condens. Matter 14 L369

[12] Scalapino D J 1995 Phys. Rep. 250329

Moriya T and Ueda K 2000 Adv. Phys. 49555

[13] Takimoto T 2000 Phys. Rev. B 62 R14641

[14] Takeuchi T et al. 2001 J. Phys. Soc. Jpn. 70877

Shishido H et al. 2002 J. Phys. Soc. Jpn. 71162

[15] Hotta T and Ueda K 2002 cond-mat/0211467 